\title{
Developmental Perspectives on Risk and Vulnerability in Alcoholic Families
}

\author{
Kenneth E. Leonard, Rina Das Eiden, Maria M. Wong, Robert A. Zucker, Leon I. Puttler, Hiram E. Fitzgerald, Andrea Hussong,
} Laurie Chassin, and Pamela Mudar

\begin{abstract}
A DEVELOPMENTAL PERSPECTIVE of maladaptation among children of alcoholics considers multiple transacting risk and protective factors to predict both adaptive and maladaptive outcomes over time. This perspective is useful for understanding both psychosocial dysfunction and risk for subsequent alcoholism among high-risk individuals. Moreover, this approach argues that understanding maladaptation among children within one developmental period is advanced particularly by longitudinal assessment of development across developmental periods or transitions. The four articles in this symposium reported on longitudinal studies of the adaptation of children of alcoholics across developmental transitions.
\end{abstract}

\section{Infants of Alcoholic Fathers: Family Context, Parenting, and Attachment}

Eiden and Leonard reported on the first wave of a multi-method longitudinal study designed to examine the impact of a father's alcoholism on parenting and infant development. This study involved a community sample of alcoholic fathers and light- to heavy-drinking mothers $(n=$

From the Department of Psychiatry(K.E.L.), State University of New York at Buffalo; Research Institute on Addictions (K.E.L., R.D.E., P.M.), Department of Psychiatry (M.M.W., R.A.Z., L.I.P.), Alcohol Research Center (M.M.W., R.A.Z., L.I.P.), and Department of Psychology (R.A.Z.), University of Michigan, Ann Arbor, Michigan; Department of Psychology (H.E.F.), Michigan State University, East Lansing, Michigan; Department of Psychology (A.H.), University of North Carolina at Chapel Hill, North Carolina; Department of Psychology (L.C.), Arizona State University, Tempe, Arizona.

Received for publication September 20, 1999; accepted December 1, 1999.

This article represents the proceedings of a symposium at the 1999 RSA Meeting in Santa Barbara, California. The Organizer/Chair was Kenneth E. Leonard. The presentations were: (1) Infants of Alcoholic Fathers: Family Context, Parenting, and Attachment by Rina Das Eiden; (2) Parent Psychopathology and the Developmental Trajectories of Internalizing and Externalizing Symptomatology in Children of Alcoholics by Maria M. Wong; (3) Adolescent Children of Alcoholics, Substance Use, and Negotiating the Leaving Home Transition by Andrea Hussong; and (4) Reductions in Drinking Over the First Year of Marriage: The Moderating Influence of Familial Alcoholism and Other Risk Factors, by Kenneth E. Leonard.

The research presented in these symposia was supported by the National Institute of Alcohol Abuse and Alcoholism (R01-AA09922, R01AA10042-04 to K.E.L.), (2R01-AA07065 to R.A.Z.) and the National Institute on Drug Abuse (DA-05227 to L.C.)

Reprint requests: Kenneth E. Leonard, Ph.D., Research Institute on Addictions, 1021 Main Street, Buffalo, New York, 14203; Fax: 716-887-2510; E-mail:leonard@ria.org

Copyright $\odot 2000$ by the Research Society on Alcoholism.
120) compared with a control sample matched with respect to mother's age, education, race, number of children, and the gender of the child. Self report measures of parental characteristics (including drinking behavior, depression, antisocial behaviors, marital conflict, and violence), infant characteristics (temperament), behavioral observations of parenting behavior, and parent-child attachment were first assessed in these families when their child was 12 months of age. Results indicated that the fathers' alcoholism was associated with higher levels of paternal and maternal antisocial behavior and depression, and partner aggression. Alcoholic fathers reported higher aggravation toward their infants and perceived their infants as being more difficult. Furthermore, the fathers' alcoholism was associated with more problematic parenting behaviors during free play, with alcoholic fathers showing less sensitivity, higher negative affect, lower positive engagement, and lower amount and quality of verbalizations. Mothers with alcoholic partners also have lower quality and frequency of verbalizations toward their infants. Furthermore, the fathers' alcoholism was associated with two high-risk patterns of mother-infant and father-infant attachment: infant avoidance and infant disorganization (as assessed by the Strange Situation paradigm). The association between the fathers' alcoholism and these aspects of parenting and parent-infant relationships seemed to be partially mediated by the fathers' depression and family aggression. In summary, the fathers' alcoholism may have an impact on family functioning and parenting as early as infancy and these effects may be of importance in understanding the risk for maladaptive developmental trajectories among children of alcoholic fathers.

Parent Psychopathology and the Developmental Trajectories of Internalizing and Externalizing Symptomatology in Children of Alcoholics

Wong, Zucker, Puttler, and Fitzgerald examined: (1) the relationship between individual differences in developmental trajectories of internalizing behavior and individual differences in developmental trajectories of externalizing behavior, and (2) how parent psychopathology affected both internalizing and externalizing problems. The data came from the University of Michigan-Michigan State University Longitudinal Study. A community sample of alcoholic men, their families, and control families were recruited. A 
total of 177 boys and their biological parents provided information in all 3 waves of the study (T1: 3-5 years old, T2: 6,8 years old, T3: 9-11 years old). Latent growth curve modeis were used to analyze the data. Results indicated that for beth internalizing and externalizing symptomatology, there were substantial interindividual differences in the starting point and the rate of change over time. On the average, internalizing behavior increased over time and externalizing behavior decreased over time. At T1, children who were high on internalizing behavior had higher scores on externalizing behavior. Over time, children who had a greater increase in internalizing behavior showed a smaller decrease in externalizing behavior. When compared with other childrent those with depressed fathers had a higher initial level of internalizing behavior. However, children with depressed mothers showed a more rapid rate of increase on internalizing behavior over time than other children. Moreover, children with alcoholic fathers had a higher initial level of externalizing behavior than other children. So did those with antisocial mothers. When compared with other children, children whose fathers were alcoholic showed a rate of decrease in externalizing behavior over time. However, at T3, their externalizing behavior scores were still higher than other children. The results suggested that (1) changes in internalizing and externalizing behavior over time are closely related to one another, and (2) paternal and maternal psychopathology have different effects on the development of childhood behavior problems.

\section{Adolescent Children of Alcoholics, Substance Use, and Negotiating the Leaving Home Transition}

Hussong and Chassin examined whether children of alcoholics (COAs) are more likely than their peers to demonstrate difficulties in negotiating the leaving home transition. A community sample of adolescents and their parents were assessed at three annual interviews (1989-1991) and a fourth interview 5 years later (initial $n=454$ ). Comparisons were made between families in which either the mother or father had a lifetime diagnosis of alcoholism and nonalcoholic families matched with respect to ethnicity, family structure, socioeconomic status, and the adolescent's age and sex. Results indicated that COAs first left the home at a younger age than their peers, and experienced more conflict and less agreement with their parents in negotiating this transition. After this first transition, young adult COAs had fewer positive and more negative feelings about the transition and, according to their parents, experienced greater feelings of abandonment by their parents than did their peers. Finally, COAs were more likely than their peers to return home after this first attempt to leave. The difficulty of this transition seems to be uniquely related to the risk of having an alcoholic parent, with few unique and consistent risks associated with having antisocial or depressed parents. One factor that partly accounts for why parent alcoholism is associated with difficulty in the leaving home transition is a greater likelihood of heavy alcohol use during middle to late adolescence among COAs compared with their peers. The results suggest a cumulative effect of developmental risks associated with parent alcoholism such that alcohol-related problems during adolescence create more difficulty during the normative transition into young adulthood.

\section{The Impact of Parental Alcoholism and Psychosocial Factors on Drinking Over the Transition to Marriage}

Leonard and Mudar reported preliminary findings from a longitudinal study of drinking from the time of marriage through the second anniversary. The marital transition is one of the key developmental events that punctuate the transition from adolescence to adulthood. At the individual level, there is often a marked shift away from more individualistic values and toward more interdependent and socially positive values over this transition. At the couple, family, and peer level, there are changes that involve the establishment of a mutually satisfactory relationship and the re-establishment or redefinition of ties, both as individuals and as a couple, with each member's extended family and peer network. Not only is marriage an important transition generally, it also seems to be a vital transition with respect to alcohol use and alcohol problems, with significant reductions in drinking and drinking problems and shifts in drinking contexts over this period. Approximately 260 couples were assessed at the time of marriage and reassessed 1 year later. After controlling for time 1 drinking, the husband's time 2 drinking was predicted by his wife's time 1 drinking and his alcohol expectancies. For wives, time 2 drinking was predicted by her husband's time 1 drinking and by her history of antisocial behavior. In addition, the predictors of time 2 drinking differed depending on the age of the subjects. Older husbands were heavier drinkers at time 2 if they scored higher on depression at time 1. Younger husbands were heavier drinkers at time 2 if they reported low relationship quality at time 1 . The drinking of younger wives was also predicted by marital quality, but the drinking of older wives was predicted by their husbands' drinking. Finally, husbands and wives with a family history of alcoholism were more strongly influenced by a heavy drinking partner than husbands and wives without such a family history. These results suggest that the risk factors for continued drinking over the transition to marriage differ for older and younger individuals and for men and women.

\section{DISCUSSION}

Zucker noted the sea change relating to a longitudinal/ developmental understanding of risk for alcohol problems that this symposium represented. While some earlier RSA meetings have provided data which spottily addressed the early emergence of risk for alcohol use disorders, these 
contributions, when mapped onto each other from an age perspective, travel all the way from infancy to early adulthood. Thus, they mark the field's research maturity, because the capacity now exists to begin to examine the processes that sustain or weaken risk variation across the entire age span between infancy and early adulthood. In a related vein, the work reported here reiterated another point that is central to a developmental understanding of process. Apparent behavioral (or trait) continuity over long age spans is usually the result of intermediary mechanisms that create the continuity. The observed apparent momentum is, in reality, the result of intermediary events which change the underlying structure of the behavior and eventually produce the polished behavioral outcome of adulthood. This is what developmentalists call epigenesis, and the speakers provided a number of examples of intermediary mechanisms that may be the ultimate mediators producing the damaged clinical endpoints of late adolescence and early adulthood.

To put this work in larger context, one of the most important developmental studies of the last several years has been the Dunedin project (Caspi et al., 1996) which has established the predictability of an alcohol dependence outcome, based upon the presence of behavioral undercontrol at age 3. A developmentally naive view of that work would attribute all the across-time continuity to genetic continuity. The present work deconstructs that conclusion, and suggests that a number of mediators may be operating to sustain the undercontrol, including: (a) the early socialization structure (Das Eiden and Leonard); (b) the presence and concurrent influence of internalizing symptomatology (Wong, Zucker, Puttler, and Fitzgerald); (c) the timing and manner in which adolescents negotiate the leaving-home transition (Hussong and Chassin); and (d) the impact of spousal behavior during early adulthood (Leonard and Mudar). The studies also illustrated that the mediational links are not always direct. To give one example from the Hussong and Chassin paper, parental alcoholism influences the onset and level of adolescent heavy alcohol use, which in turn increases the likelihood of leaving home to escape unhappiness, to find solace in a romantic relationship, but simultaneously decreases the choice to pursue educational and occupational opportunities.

At the same time, some of these studies raise the question about how much of the apparently mediated, socialinteractional variance can be regarded as indirect effects of the genetic variance (which supports both the expression of behavioral undercontrol in the high-risk offspring and in their biologically related caregivers). Both the Das Eiden and Hussong studies implicated poor parental control structures as mediators of risky child outcome; at this time we do not know how far back we should extend this casual chain. Ultimately, the upstream links will need to be examined

More generally, these studies addressed the continuity of process issues, and specification of mechanism, at a level of precision that simply did not exist even 5 years ago. As the studies progress, our understanding of the mediational structure and development of early risk factors for alcohol problems will become increasingly more precise, and along with that, our capacity to mount increasingly more focused preventive interventions will also emerge.

\section{REFERENCES}

Caspi A, Moffitt TE, Newman DL, Silva EA (1996) Behavioral observations at age 3 years predict adult psychiatric disorders: Longitudinal evidence from a birth cohort. Arch Gen Psychiatry 53:1033-1039. 